\title{
Investigation and Macroscopic Anatomical Study of Referred Pain in Patients with Hip Disease
}

\author{
Junya Sakamoto, PhD, $\mathrm{PT}^{1)^{*}}$, Yosuke Morimoto, $\mathrm{PT}^{1,2)}$, Shun Ishit, $\mathrm{PT}^{1)}$, \\ Jiro Nakano, PhD, PT $^{3)}$, Yoshitaka Manabe, PhD, MD ${ }^{4}$, Minoru Okita, PhD, PT $^{2}$, \\ Toshiyuki Tsurumoto, $\mathrm{PhD}, \mathrm{MD}^{5)}$ \\ 1) Department of Rehabilitation, Nagasaki University Hospital: 1-7-1 Sakamoto, Nagasaki 852-8501, \\ Japan \\ 2) Department of Locomotive Rehabilitation Science, Unit of Rehabilitation Sciences, Nagasaki Univer- \\ sity Graduate School of Biomedical Sciences, Japan \\ 3) Department of Physical Therapy Science, Unit of Physical and Occupational Therapy, Nagasaki \\ University Graduate School of Biomedical Sciences, Japan \\ 4) Department of Oral Anatomy and Dental Anthropology, Unit of Basic Medical Sciences, Nagasaki \\ University Graduate School of Biomedical Sciences, Japan \\ 5) Department of Macroscopic Morphology, Unit of Basic Medical Sciences, Nagasaki University \\ Graduate School of Biomedical Sciences, Japan
}

\begin{abstract}
Purpose] The aim of this study was to examine the incidence and patterns of referred pain in patients with hip disease, as well as the nerve distribution in the hip and knee joints of 2 cadavers. [Subjects and Methods] A total of 113 patients with hip joint disease were included in the investigation. The incidence of regional pain and referred pain patterns were evaluated before and after arthroplasty. Two cadavers were macroscopically observed to verify the nerve innervation of the hip and knee joints. [Results] Anterior knee pain was observed preoperatively in $13.3 \%$ (in resting) and $33.6 \%$ (in motion) of the patients, which was comparable with the incidence of greater trochanter pain. In addition, the preoperative incidence rates of knee pain in resting and motion markedly decreased postoperatively. Of note is the remarkable incidence of pain radiating to the ventral lower limb. An anteromedial innervation was determined in the cadavers by the articular branches of the obturator and femoral nerve, which supply small branches to the knee joints. [Conclusion] Our results suggest that the distribution of the incidence of pain among the patients with hip disease is diverse owing to the sensory distribution of the femoral and obturator nerves.

Key words: Hip joint disease, Referred pain, Macroscopic anatomy
\end{abstract}

(This article was submitted Jul. 8, 2013, and was accepted Aug. 28, 2013)

\section{INTRODUCTION}

Pain is the main symptom of hip joint diseases such as osteoarthritis (OA) and osteonecrosis. Clinical presentation is dominated by pain during rest and joint motion, which results in loss of motion function and limitation in activities of daily living (ADL). A previous study reported that physical therapy as a conservative therapy reduced pain in OA patients ${ }^{1}$. However, physical therapy can only delay the progress of hip OA. In contrast, in end-stage OA, total hip arthroplasty (THA) is considered to be one of the most successful joint surgeries for the alleviation of prolonged pain in patients with hip disease. In fact, Kawada et al. ${ }^{2}$ reported that pain in the hip joint provided motivation for patients

*Corresponding author. Junya Sakamoto (E-mail: jun-saka@, nagasaki-u.ac.jp)

(C2014 The Society of Physical Therapy Science. Published by IPEC Inc. This is an open-access article distributed under the terms of the Creative Commons Attribution Non-Commercial No Derivatives (by-ncnd) License $<$ http://creativecommons.org/licenses/by-nc-nd/3.0/>. with hip disease to undergo arthroplasty. Moreover, preoperative pain in resting and motion are factors largely influencing the occurrence of pain following total knee arthroplasty $^{3)}$. Therefore, for a physical therapist managing pain, it is important to correctly perceive the reality of pain in patients with hip disease.

In clinical settings, patients with hip joint disease sometimes present with complaints of pain around not only the hip joint but also the thigh, knee, and lower leg. In the literature, the groin, thigh, and knee are reported as the reference regions of patients with hip disease ${ }^{4,5}$. Hip joint pain in OA has been classically described as presenting in the groin, with reference to the area below the knee. Khan et al. ${ }^{4)}$ reported that $47 \%$ of patients with hip OA had pain below the knee. Morimoto et al. ${ }^{6}$ ) reported that $34.6 \%$ of patients awaiting arthroplasty have pain below the knee, even though they did not have lower limb or lumbar diseases.

Pain referred from one part of the body to another is called referred pain. The mechanisms of referred pain have not yet been elucidated. Ruch ${ }^{7}$ hypothesized that referred pain originates from convergence and projection; somatic 
and visceral fibers from each of the primary neurons converging upon one dorsal horn neuron in the spinal cord. Sinclair et al. ${ }^{8)}$ proposed that referred pain is caused by primary afferent fibers bifurcating into somatic and visceral structures. These hypotheses mean that primary sensory neurons relate to the occurrence of referred pain.

In earlier literatures, the source of pain radiating from the hip to below the knee was considered to be related to the femoral and obturator nerves, which supply articular branches to the hip joint and cutaneous branches to the thigh and around the knee ${ }^{9,10)}$. Khan and Woolson ${ }^{9)}$ reported that referred pain in hip OA was transported along the saphenous branch of the femoral nerve, which is responsible for the sensory innervation of the anterior and anterolateral regions of the hip joint capsule. The saphenous nerve, a branch of the femoral nerve, innervates the cutaneous anterior and medial knee joints. Therefore, the femoral nerve is believed to contribute to the referred pain originating from the hip joint. The obturator nerves supply the articular branches to the anteromedial section of the hip joint capsule. In addition, these nerves innervate the anterior and medial aspects of the thigh and knee. A previous study reported that blockade of these nerves by intra-articular injection reduced hip referral pain in patients with hip $\mathrm{OA}^{11)}$, indicating that referred pain to the knee of patients with hip disease is transmitted by the femoral and obturator nerve, which innervates the anterior medial and lateral aspects of the hip joint. In our clinical experience, pain along the region of the femoral and obturator nerves is often preoperatively found in patients with hip disease, especially in motion, e.g., standing up or walking, rather than in resting. However, this pain disappears postoperatively in most cases. We believe that most cases of referred pain, especially anterior knee pain, are referred from the hip joint, with the articular branches of the femoral and obturator nerves being related to the pain. However, we have been unable to find no evidence supporting our opinion. In addition, only a few early reports have described the involvement of the articular branches of these nerves in hip and knee pain.

In this study, we investigated the frequency of regional and referred pain patterns in patients with hip disease at pre- and post-arthroplasty. In addition, we observed two adult cadavers to analyze the nerve supply of the hip and knee joints from the femoral and obturator nerves.

\section{SUBJECTS AND METHODS}

Ethical approval for this investigation was granted by the Nagasaki University Hospital Ethics Board (No. 13040158). The medical records of patients with hip joint disease who underwent total hip arthroplasty at Nagasaki University Hospital between April 2008 and November 2011 were retrospectively reviewed. Patients with dementia, diseases of the lower limbs, and lumbar disease were excluded. The background data of 113 patients are summarized in Table 1. Of the patients, 92 were women and 21 were men, with a mean age of $63.2 \pm 11.4$ years (range, $39-84$ years). The subjects' mean height, weight, and body mass index were $154.7 \pm 7.9 \mathrm{~cm}, 56.8 \pm 10.5 \mathrm{~kg}$, and $23.8 \pm 3.8 \mathrm{~kg} / \mathrm{m}^{2}$, respec-
Table 1. Summary of the subjects' background data

\begin{tabular}{lc}
\hline Age (years) & $63.2 \pm 11.4$ \\
Sex & \\
Female & 92 \\
Male & 21 \\
Height $(\mathrm{cm})$ & $154.7 \pm 7.9$ \\
Weight $(\mathrm{kg})$ & $56.8 \pm 10.5$ \\
BMI $\left(\mathrm{kg} / \mathrm{m}^{2}\right)$ & $23.8 \pm 3.8$ \\
Diagnosis & 100 \\
Hip OA & 13 \\
Osteonecrosis & 107 \\
Operation & 5 \\
THA & 1 \\
BHA & $6.9 \pm 8.2$ \\
BHR \\
Duration of pain (years) \\
\hline The data are expressed as mean \pm SD, unless other- \\
wise specified
\end{tabular}

tively. The documented diagnoses were as follows: hip OA $(n=100)$ and osteonecrosis of the femoral head $(n=13)$. The scheduled arthroplastic procedures were THA $(\mathrm{n}=107)$, bipolar hip arthroplasty (BHA, $\mathrm{n}=5$ ), and Birmingham hip resurfacing $(\mathrm{BHR}, \mathrm{n}=1)$. Furthermore, the mean duration of continuing pain was $6.9 \pm 8.2$ years.

For regional pain assessment, based on a previous study ${ }^{2)}$, we referred to a body chart that was used as part of physical therapy evaluation. On the body chart, which depicted the area from the lower limb to the foot, all the patients were asked to mark the areas of the limb that experienced pain in resting and motion. They were allowed to mark several regions on the chart. The evaluation was conducted before ( $1.4 \pm 0.9$ days) and after the operation ( $15.0 \pm$ 5.0 days). For analysis of regional pain frequency and pain patterns, the pain region was divided into the groin, greater trochanter, glutealis, thigh (anterior, posterior, lateral, and medial), knee (anterior and posterior), and below the leg.

Two formalin-preserved adult Japanese cadavers were subjected to macroscopic observation. All the cadavers were supplied by body donation, and consent for their use for education and research had been given. Given that no data allowing the identification of the individuals were presented, the publication of this study does not pose any ethical problems. The femoral and obturator nerves and their branches were exposed, and the articular branches were studied macroscopically. The findings were photographed.

\section{RESULTS}

The incidence of regional pain in the 113 patients is summarized in Table 2. We found that 38 patients $(33.6 \%)$ in resting and 71 patients $(62.8 \%)$ in motion preoperatively complained of groin pain, which had the highest incidence rate. In order of decrease, it was followed by pain in the greater trochanter (21 patients [18.6\%] in resting and 39 patients $[34.5 \%]$ in motion) and glutealis (21 patients [18.6\%] 
Table 2. The incidence of the regional pain patterns

\begin{tabular}{ccccccccccc}
\hline & groin & $\begin{array}{c}\text { greater } \\
\text { trochanter }\end{array}$ & glutealis & $\begin{array}{c}\text { thigh } \\
\text { (anterior) }\end{array}$ & $\begin{array}{c}\text { thigh } \\
\text { (posterior) }\end{array}$ & $\begin{array}{c}\text { thigh } \\
\text { (lateral) }\end{array}$ & $\begin{array}{c}\text { thigh } \\
\text { (medial) }\end{array}$ & $\begin{array}{c}\text { knee } \\
\text { (anterior) }\end{array}$ & $\begin{array}{c}\text { knee } \\
\text { (posterior) }\end{array}$ & $\begin{array}{c}\text { below } \\
\text { the leg }\end{array}$ \\
\hline $\begin{array}{c}\text { Rest } \\
\text { pre }\end{array}$ & $38(33.6)$ & $21(18.6)$ & $21(18.6)$ & $12(10.6)$ & $4(3.5)$ & $13(11.5)$ & $5(4.4)$ & $15(13.3)$ & $1(0.9)$ & $9(8.0)$ \\
post & $17(15.0)$ & $13(11.5)$ & $13(11.5)$ & $5(4.4)$ & $4(3.5)$ & $5(4.4)$ & $6(5.3)$ & $7(6.2)$ & $5(4.4)$ & $6(5.3)$ \\
$\begin{array}{c}\text { Motion } \\
\text { pre }\end{array}$ & $71(62.8)$ & $39(34.5)$ & $42(37.2)$ & $22(19.5)$ & $11(9.7)$ & $21(18.6)$ & $4(3.5)$ & $38(33.6)$ & $10(8.8)$ & $14(12.4)$ \\
post & $37(32.7)$ & $38(33.6)$ & $23(20.4)$ & $22(19.5)$ & $10(8.8)$ & $20(17.7)$ & $15(13.3)$ & $19(16.8)$ & $8(7.1)$ & $8(7.1)$ \\
\hline
\end{tabular}

The data are expressed as number of cases (\%). pre, preoperative; post, postoperative

Table 3. Changes in the incidence of knee pain from preoperation to postoperation

\begin{tabular}{lrcrc}
\hline & \multicolumn{2}{c}{ Resting } & \multicolumn{2}{c}{ Motion } \\
\cline { 2 - 5 } & pre & post & pre & post \\
\hline Anterior & 15 & 1 & 38 & 12 \\
Posterior & 1 & 1 & 8 & 2 \\
\hline
\end{tabular}

Pre, preoperative; post, postoperative

in resting and 42 [37.2\%] in motion). Anterior and lateral thigh pains occurred in $12(10.6 \%)$ and 13 patients $(11.5 \%)$ in resting, and $22(19.5 \%)$ and 21 patients $(18.6 \%)$ in motion, respectively. Anterior knee pain was preoperatively experienced by 15 patients $(13.3 \%)$ in resting and 38 patients $(33.6 \%)$ in motion. At postoperation, most cases of regional pain had improved in resting and motion. Groin pain had the highest incidence in resting (17 cases, $15.0 \%)$ and second highest in motion (37 cases, 32.7\%). Greater trochanter and glutealis pains occurred in 13 cases $(11.5 \%)$ in resting and $38(33.6 \%)$ and 23 cases (20.4\%) in motion, respectively. The postoperative thigh pain frequency was comparable with or better than that before the operation. Anterior knee pain was recorded for 7 cases $(6.2 \%)$ in resting and 19 cases $(16.8 \%)$ in motion.

Table 3 presents the changes in the incidence of knee pain from before to after the operation. Fifteen patients $(13.3 \%)$ in resting and 42 patients $(37.2 \%)$ in motion preoperatively complained of knee pain. At postoperation, anterior knee pain disappeared in 14 patients in resting and 26 patients in motion.

Twelve pain referred patterns are presented in Table 4 . Most of the referred pain patterns were observed in the ventral aspect of the lower limb. At preoperation, the highest incidence of referred pain pattern in resting was observed in the groin alone (12 cases, $10.6 \%$ ), followed by groin pain referred to the thigh and knee (11 cases, 9.7\%). In motion, however, the incidence of groin pain referred to the thigh and knee increased compared with that in resting. At postoperation, the referred pain patterns in resting and motion decreased compared with those at preoperation.

We observed two adult cadavers to analyze the nerve supply to the hip and knee joints. In one case, a small branch ascended vertically from the branch of the femoral nerve to the pectineal muscle and entered to the anteromedial side of the iliofemoral ligament (Fig. 1a, b). In the same case, other minor branches of the femoral nerve that pierced the vastus medialis entered the joint capsule from the superior medial aspect of the patella (Fig. 1c, d). In the other case, a small branch from the posterior branch of the obturator nerve entered the anteromedial aspect of the pubofemoral ligament (Fig. 2a, b). In addition, the branch derived from the posterior branch of the obturator nerve ran down along the adductor longus, following convergence with the saphena nerve (Fig. 2c, d). The small branch entered the knee joint from the medial aspect of the patella (Fig. 2e).

\section{DISCUSSION}

Several studies have reported that pain originating from the hip joint referred to the thigh, knee, and the below lower leg in patients with hip disease ${ }^{4-6,9)}$. Among these regions, the anterior knee is the most common pain location to which hip joint and anterior thigh pain radiate. The saphenous branch of the femoral nerves that supply articular branches to the hip joint has been considered to be a source of referred pain originating from the hip joint. However, only a few previous research studies have reported the occurrence patterns of referred pain with consideration of the neuroanatomical aspect of the lower limb. In this study, we retrospectively investigated the preoperative and postoperative incidences of regional pain, and occurrence patterns of referred pain. In addition, we observed the sensory nerve innervation of the hip and knee joints in two cadavers, using a macroscopic approach.

At preoperation, the most common pain locations in the patients with hip disease were the groin, greater trochanter, glutealis, anterior thigh, and anterior knee. The incidence rates of these regional pains in motion were markedly increased compared with those in resting. The incidence of anterior knee pain was comparable with that of greater trochanter pain. Khan and Woolson $\left.{ }^{9}\right)$ reported that $65 \%$ of patients with primary hip disease had pain in the knee area, $88 \%$ of whom had anterior knee pain. Hsieh et al. ${ }^{5)}$ noted that anterior knee pain occurred in $35.4 \%$ of patients with end-stage hip disease before THA. Our findings regarding the incidence of anterior knee pain support those of previous studies. In contrast to our findings, , Lesher et al. ${ }^{12)}$ reported that only $2 \%$ of the patients who underwent diagnostic and potentially therapeutic fluoroscopically guided intra-articular hip injections had knee pain. The difference in the incidence of knee pain may arise from the disease 
Table 4. The incidence of the 12 observed patterns of pain

\begin{tabular}{lcccc}
\hline & \multicolumn{2}{c}{ Rest } & \multicolumn{2}{c}{ Motion } \\
\hline Pain patterns & pre & post & pre & post \\
\hline Groin alone & $12(10.6)$ & $6(5.3)$ & $17(15.0)$ & $9(8.0)$ \\
Greater trochanter alone & $1(0.9)$ & $4(3.5)$ & $2(1.8)$ & $3(2.7)$ \\
Groin + greater trochanter & $3(2.7)$ & $3(2.7)$ & $2(1.8)$ & $4(3.5)$ \\
Groin + glutealis & $7(6.2)$ & $1(0.9)$ & $9(8.0)$ & $4(3.5)$ \\
Groin + thigh (anterior and medial) & $11(9.7)$ & $7(6.2)$ & $18(15.9)$ & $17(15.0)$ \\
Groin + thigh (anterior and medial) + Knee (anterior) & $6(5.3)$ & $1(0.9)$ & $12(10.6)$ & $7(6.2)$ \\
Groin + knee (anterior) & $6(5.3)$ & $2(1.8)$ & $21(18.5)$ & $8(7.1)$ \\
Glutealis alone & $5(4.4)$ & $3(2.7)$ & $8(7.1)$ & $11(9.7)$ \\
Glutealis + thigh (posterior) & $2(1.8)$ & $1(0.9)$ & $4(3.5)$ & $2(1.8)$ \\
Glutealis + thigh (posterior) + Knee (posterior) & $1(0.9)$ & $0(0.0)$ & $1(0.9)$ & $0(0.0)$ \\
Glutealis + knee (posterior) & $0(0.0)$ & $2(1.8)$ & $3(2.7)$ & $3(2.7)$ \\
Thigh and/or knee & $5(4.4)$ & $7(6.2)$ & $9(8.0)$ & $20(17.7)$ \\
\hline
\end{tabular}

The data are expressed as number of cases (\%). Pre, preoperative; post, postoperative

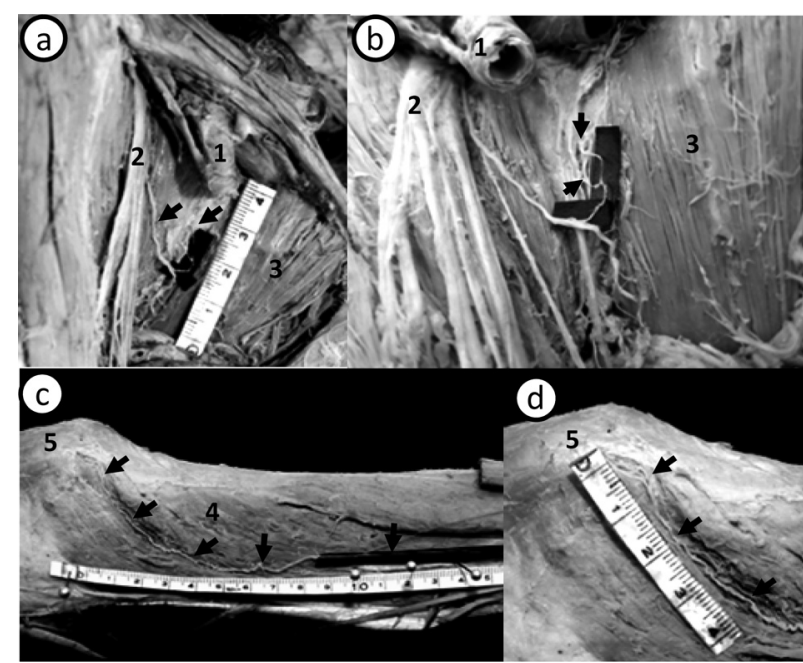

Fig. 1. Small branches of the femoral nerve in the hip and knee joint. $(a, b)$ The anteromedial aspect of the right hip joint. (c, d) The medial aspect of the right knee joint. 1: femoral artery. 2: femoral nerve. 3: pectineal muscle. 4: vastus medialis. 5: patella. In one cadaver, a small branch (arrow) that entered the anterior side of the iliofemoral ligament originated from the femoral nerve (a, b). In the same cadaver, one minor muscular branch that pierced the vastus medialis entered the joint capsule from the superior medial aspect of the patella (c, d). It seems that each branch is an articular branch of the femoral nerve innervating the hip and knee joints.

stage of subjects. Our subjects were patients with end-stage hip joint disease who underwent arthroplasty as were the subjects of study by Khan and Woolson ${ }^{9)}$. There is a possibility that stage of hip joint disease may affect the incidence of regional pain. At postoperation, the incidence rates of almost all regional pain patterns in resting and motion were reduced compared with those at preoperation. We speculate that these referred pains originated in hip joint, consider-

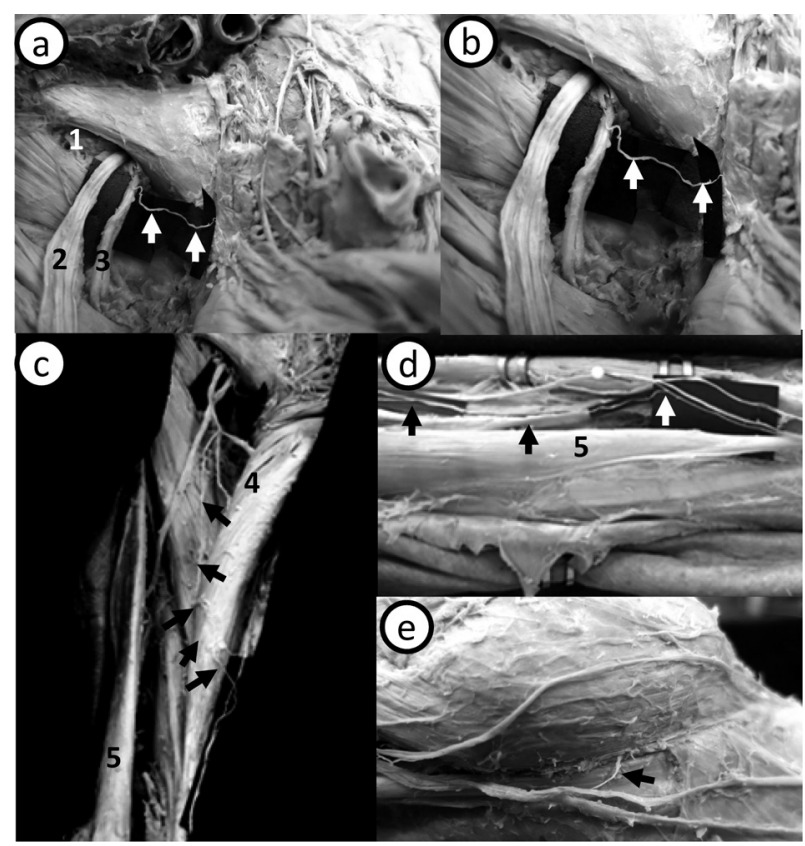

Fig. 2. Small branches of the obturator nerve in the hip and knee joint $(a, b)$ The anteromedial aspect of the left hip joint. (c) The anteromedial aspect of the proximal left thigh. (d) The medial aspect of the distal left thigh (around the adductor canal). (e) The medial aspect of the left knee joint. 1: obturator foramen. 2: anterior branch of the obturator nerve. 3: posterior branch of the obturator nerve. 4: adductor longus. 5: gracilis. In the other cadaver, a small branch (arrow) originated from the posterior branch of the obturator nerve. It entered in the anteromedial aspect of the pubofemoral ligament (a). The enlarged section is shown in (b). A branch (arrow) of the posterior branch of the obturator nerve ran down along the adductor longus (c), converging with the saphena nerve (arrow in e). The small branch (arrow) entered the knee joint from the medial aspect of the patella (e). 
ing that the patients with lower limb and lumbar diseases were excluded from this study. Therefore, if referred pain from the hip joint induces preoperative anterior knee pain, we can infer that arthroplasty, which removes pain-causing tissues, reduces the anterior knee pain. Of our cases, 15 had anterior knee pain in resting at preoperation, which disappeared in 14 patients $(93.3 \%)$ at postoperation. Moreover, in $26(68.4 \%)$ of 38 patients, anterior knee pain in motion disappeared postoperatively. These results suggest that most referred pain radiate from the hip joint to the knee in the patients with hip disease before hip joint arthroplasty.

The incidence of regional pain among our patients indicated that the most common pain locations were the groin, anterior knee, and ventral lower limb. These areas are innervated by the femoral and obturator nerves that supply articular branches to the hip joint. We speculated that the referred pain pattern in hip joint disease occurs along areas innervated by these nerves. We identified 12 referral pain patterns based on body image maps. In the preoperative period, in referred pain pattern in resting was observed most commonly in the groin alone, and groin pain with an anterior thigh referral had an incidence rate of approximately $10 \%$. Meanwhile, the pain pattern in motion was observed to be in the groin, with thigh and/or anterior knee referral. These referred pain patterns decreased postoperatively, except in the groin with a thigh pain pattern in motion. Birnbaum et al. ${ }^{10)}$ suggested that the anterior section of the hip joint capsule is supplied by the articular branches of the femoral and obturator nerves. Moreover, the cutaneous branches of these nerves innervate the anterior and medial aspects of the thigh. Therefore, it is likely that the femoral and obturator nerves are the source of the referred pain pattern observed in the ventral lower limb. In addition, Hirasawa et al. ${ }^{13)}$ reported that the branches of these nerves enter the anterosuperior or medial aspect of the knee joint, which is thought to be the causative factor of knee pain originating from the hip joint.

The femoral and obturator nerves, derived from lumbar nerve roots 2 to 4 , have great influence on the hip joint capsule, including the sensory dermatomes of the groin, medial and anterior thigh, anterior knee, and medial leg. To our knowledge, this is the first investigation to observe the branches entering the hip and knee joints at the same time. In one cadaver, the femoral nerve supplied a small branch to the anterior side of the iliofemoral ligament and superior medial aspect of the patella penetrating the vastus medialis. In the other cadaver, the obturator nerve supplied a small branch to the anteromedial aspect of the pubofemoral ligament and the medial aspect of the patella. Based on the studies of Birnbaum et al. ${ }^{10)}$ and Hirasawa et al. ${ }^{13)}$, these small branches seemed to be articular branches of the femoral and obturator nerves. In addition, Hirasawa et al. ${ }^{13)}$ found the branches, divided from the nerves innervated to the joint capsule, were distributed to the anterior and posterior cruciate ligaments. Moreover, Miura et al. ${ }^{14)}$ reported that approximately $1.6 \%$ of all DRG neurons innervating the hip joints had dichotomizing axons that extended to the medial portion of knee skin, which may play some role in the occurrence of referred pain. In this regard, our findings suggest that the articular branches of the femoral and obturator nerves contributed to the referred pain in the patients with hip joint disease.

It should be noted that anterior knee pain postoperatively remained in 12 patients, contradicting our aforementioned opinion. Kawada et al. ${ }^{2}$ reported that a few patients with hip joint disease were successfully treated with nerve root block therapy to reduce the referred pain, although an intraarticular block injection therapy failed to reduce the pain. This finding suggests that the sensitization of spinal cord neurons caused by a prolonged noxious stimulus occurred in the patients with hip disease. In general, patients with hip OA have chronic pain derived from degenerative changes caused by bone deformation and synovial inflammation. Therefore, the sensitization of spinal cord neurons caused by a noxious stimulus may be considered as a mechanism underlying the persistence of referred pain after arthroplasty. However, a recent study indicated that immobilization of the hind limbs of rats induced chronic pain ${ }^{15}$ ). A previous study assessing pain after THA reported that poor preoperative function might affect recovery unfavorably, which might in turn lead to chronic pain ${ }^{16)}$. Continuous pain causes a small amount of physical exertion. Therefore, chronic pain in OA patients leads to a reduction in the amount of physical activity, which may lead to another chronic pain pattern. The present study was unable to clarify the details of the pain origin in the cases presented. However, future examination of these points is necessary.

This study had some limitations. First, the postoperative evaluation was initiated approximately 2 weeks after arthroplasty; thus, post surgical pain may have influenced the incidence of regional pain and pain patterns, particularly, pain in the glutealis, the greater trochanter and the lateral thigh in motion. Therefore, we believe that a follow-up survey is necessary. Second, we observed only two cadavers. Nerve distribution in the hip and knee joints may have more variation. Therefore, we think that more observation is necessary to clarify the relation between referred pain in patients with hip disease and the articular branches of the femoral and obturator nerves.

\section{ACKNOWLEDGEMENT}

The authors thank Mr. Kazunobu Saiki of the Department of Gross Anatomy, Unit of Basic Medical Sciences, Nagasaki University Graduate School of Biomedical Sciences, for his assistance with the gross anatomical observation.

\section{REFERENCES}

1) Zhang W, Moskowitz RW, Nuki G, et al.: OARSi recommendations for the management of hip and knee osteoarthritis, part 1: critical appraisal of existing treatment guidelines and systematic review of current research evidence. Osteoarthritis Cartilage, 2007, 15: 981-1000. [Medline] [CrossRef]

2) Kawada $T$, Ushida $T$, Ikeuchi M, et al.: Clinical study of hip joint referred pain. Pain Res, 2006, 21: 127-132.

3) Rakel BA, Blodgett NP, Bridget Zimmerman M, et al.: Predictors of postoperative movement and resting pain following total knee replacement. Pain, 2012, 153: 2192-2203. [Medline] [CrossRef]

4) Khan AM, McLoughlin E, Giannakas K, et al.: Hip osteoarthritis: where is the pain? Ann R Coll Surg Engl, 2004, 86: 119-121. [Medline] [CrossRef] 
5) Hsieh PH, Chang Y, Chen DW, et al.: Pain distribution and response to total hip arthroplasty: a prospective observational study in 113 patients with end-stage hip disease. J Orthop Sci, 2012, 17: 213-218. [Medline] [CrossRef]

6) Morimoto M, Kondo Y, Shimosako J, et al.: Investigation of pain in hip disease patients before and after arthroplasty. J Phys Ther Sci, 2011, 23 535-538. [CrossRef]

7) Ruch TC: Visceral sensation and referred pain. In: Fulton JF, ed. Howell's textbook of physiology, 15th ed. Philadelphia: WB Saunders, 1946, pp $385-401$.

8) Sinclair DC, Weddell G, Feindel WH: Referred pain and associated phenomena. Brain, 1948, 71: 184-211. [Medline] [CrossRef]

9) Khan NQ, Woolson ST: Referral patterns of hip pain in patients undergoing total hip replacement. Orthopedics, 1998, 21: 123-126. [Medline]

10) Birnbaum K, Prescher A, Hessler S, et al.: The sensory innervation of the hip joint an anatomical study. Surg Radiol Anat, 1997, 19: 371-375. [Medline] [CrossRef]

11) Odoom JE, Allen GM, Wilson DJ: Response to local anaesthetic injection as a predictor of successful hip surgery. Clin Radiol, 1999, 54: 430-433. [Medline] [CrossRef]

12) Lesher JM, Dreyfuss P, Hager N, et al.: Hip joint pain referral patterns: descriptive study. Pain Med, 2008, 9: 22-25. [Medline] [CrossRef]

13) Hirasawa $Y$, Okajima $S$, Ohta $M$, et al.: Nerve distribution to the human knee joint: anatomical and immunohistochemical study. Int Orthop, 2000, 24: 1-4. [Medline] [CrossRef]

14) Miura $Y$, Ohtori S, Nakajima T, et al.: Dorsal root ganglion neurons with dichotomizing axons projecting to the hip joint and the knee skin in rats: possible mechanism of referred knee pain in hip joint disease. J Orthop Sci, 2011, 16: 799-804. [Medline] [CrossRef]

15) Nakano J, Sekino Y, Hamaue Y, et al.: Changes in hind paw epidermal thickness, peripheral nerve distribution and mechanical sensitivity after immobilization in rats. Physiol Res, 2012, 61: 643-647. [Medline]

16) Johansson HR, Bergschmidt P, Skripitz R, et al.: Impact of preoperative function on early postoperative outcome after total hip arthroplasty. J Orthop Surg (Hong Kong), 2010, 18: 6-10. [Medline] 